Review Article

\title{
Promoting Prognostic Model Application: A Review Based on Gliomas
}

\author{
Xisong Liang $\mathbb{D}^{1},{ }^{1}$ Zeyu Wang $\mathbb{D}^{1},{ }^{1}$ Ziyu Dai $\mathbb{D},{ }^{1}$ Hao Zhang $\mathbb{D}^{1},{ }^{1}$ Quan Cheng $\mathbb{D},{ }^{1,2,3}$ \\ and Zhixiong Liu iD 1,2 \\ ${ }^{1}$ Department of Neurosurgery, Xiangya Hospital, Central South University, Changsha 410008, China \\ ${ }^{2}$ National Clinical Research Center for Geriatric Disorders, Xiangya Hospital, Central South University, Changsha 410008, China \\ ${ }^{3}$ Department of Clinical Pharmacology, Xiangya Hospital, Central South University, Changsha 410008, China
}

Correspondence should be addressed to Quan Cheng; chengquan@csu.edu.cn and Zhixiong Liu; zhixiongliu@csu.edu.cn

Xisong Liang and Zeyu Wang contributed equally to this work.

Received 14 May 2021; Accepted 3 July 2021; Published 3 August 2021

Academic Editor: Xiangya Ding

Copyright (C) 2021 Xisong Liang et al. This is an open access article distributed under the Creative Commons Attribution License, which permits unrestricted use, distribution, and reproduction in any medium, provided the original work is properly cited.

Malignant neoplasms are characterized by poor therapeutic efficacy, high recurrence rate, and extensive metastasis, leading to short survival. Previous methods for grouping prognostic risks are based on anatomic, clinical, and pathological features that exhibit lower distinguishing capability compared with genetic signatures. The update of sequencing techniques and machine learning promotes the genetic panels-based prognostic model development, especially the RNA-panel models. Gliomas harbor the most malignant features and the poorest survival among all tumors. Currently, numerous glioma prognostic models have been reported. We systematically reviewed all 138 machine-learning-based genetic models and proposed novel criteria in assessing their quality. Besides, the biological and clinical significance of some highly overlapped glioma markers in these models were discussed. This study screened out markers with strong prognostic potential and 27 models presenting high quality. Conclusively, we comprehensively reviewed 138 prognostic models combined with glioma genetic panels and presented novel criteria for the development and assessment of clinically important prognostic models. This will guide the genetic models in cancers from laboratory-based research studies to clinical applications and improve glioma patient prognostic management.

\section{Introduction}

Malignant tumors are characterized by therapeutic resistance, frequent recurrence, and distant metastasis, which cause difficulties in treating by either surgical resection or adjunctive therapies, leading to poor prognosis. For better clinical management, many prognostic models were proposed to analyze survival [1]. Previous models with unformulated predictors stratified patients into relative risk groups. However, they did not provide quantitative results or absolute risk stratification. Machine learning algorithms can identify critical patterns through big and complex data with high accuracy [2]. Common algorithms applied in cancer prediction include weighted gene coexpression network analysis (WGCNA), L1-penalized least absolute shrinkage selection operator (LASSO), Cox proportional hazards (PH) model [3], Neural Network [2], and Elastic regression [4]. Based on these machine learning algorithms, risk scores and further pictorial nomograms are constructed for addressing this issue $[5,6]$. Risk score models are calculated with a spectrum of parameters in predicting clinical outcome risks. Samples are from local patient cases or online data. Establishing a model requires two main steps: development and validation [5]. First, predictors are selected and risks are calculated; thereafter, performance estimations are performed to assess the predictive quality. Second, the model is validated internally and externally (independent data set). Performance estimation is also performed [7].

Gliomas are common intracranial tumors causing the highest mortality rates in all cancer types [8]. Routine treatments for gliomas include surgical excision, radiotherapy, and chemotherapy. The world health organization (WHO) grouped gliomas into four grades based on their histological features: WHO grades I, II, III, and IV [9]. Low- 
grade gliomas (LGGs) comprise WHO grades I and II, showing a relatively good prognosis. High-grade gliomas (HGGs) consist of WHO grades III and IV, manifesting worse survival outcomes [9]. Histological classification provides an understanding of glioma behavior. However, molecular groups differentiate prognostic groups more accurately [10] (Figure 1). The 2016 Central Nervous System WHO classification, therefore, provided molecular features including IDH-mutant/wildtype astrocytoma and glioblastoma, IDH-mutant/wildtype, and 1p/19q-codeleted/noncodeleted oligodendroglioma, H3K27M-mutant/wildtype diffuse midline glioma, and RELA fusion-positive/negative ependymoma to precisely classify gliomas [11]. These and other markers such as CDKN2A and EGFR were combined to develop prognostic models for glioma patients [12].

The poor prognosis of gliomas triggered the development of a clinically useful and effective model to assess survival risks for subsequent therapeutic strategies. This review systematically summarized and compared all 138 risk score models for gliomas with multivariable markers (Figure 2). It also presented the clinical significance of some frequently reported predictors, thus guiding the advanced prediction models for glioma. This will be conducive to translation from laboratory-based models to clinical available tools and clinical prognosis management improvement.

\section{Rules for Evaluating Model Quality and Exclusion Criteria}

The TRIPOD statement standardized reports on the prediction models. It proposes a checklist of 22 items required in the development and validation process [13]. However, it does not judge model quality. Thus, we screened through the 138 models and proposed novel criteria that classified them into different quality groups (Figure 3); the criteria are listed in Table 1. After quality division, the high- and mediumquality models were discussed, and the low-quality group models were not presented (Table S1).

Performance estimation, validation, and EPV are vital factors to assess the model quality. Performance includes discrimination and calibration. Discrimination refers to the capability that differentiates patients from events that happen or not, which functions as the most essential quality results [14]. Calibration compares estimated event rates with observed rates [15]. Less efficacy arises when discrimination is confused with performance. Secondly, most studies lack calibration results [16]. For discrimination, we defined area under the curve (AUC), or c-index $\geq 0.80$ as high accuracy, AUC $\geq 0.70$ as acceptable, and AUC $<0.7$ as low accuracies. The repeatability and transportability of the model should be verified through internal and external validation before clinical application [15]. Proper EPV requires 10 minimally determined by rule of thumb, but when many low-prevalence predictors are included, EPV should be up to 20 [17]; otherwise, it is considered an overfitting model.

Other aspects including variable number, missing value, outcome definition, reference genome, and annotation update also contribute to the model assessment. Excessive variables increase detective costs and decrease practicability.
For most models contain less than 10 predictors and no certain conclusion have been presented, we defined less than 10 appropriate predictors. Missing data can lead to bias when not handled properly. Outcome definition is clearer and more informative with specific follow-up time than overall survival (OS). Reference genome and annotation updates can cause reversed individual risk prediction due to multiple gene expression diversity [18]. The inconsistent cutoff values between training and validation sets, screening method, and the threshold for predictors are complex questions and remain unresolved; they were not reviewed.

We excluded studies if (1) a single genetic predictor was employed with or without other types of predictors, (2) the model predicts glioma diagnosis at the time of screening, and (3) the model is inadequately presented without a regression equation or risk score.

\section{RNA Models}

3.1. mRNAs. Message RNA (mRNA) plays a critical role in central dogma that controls protein synthesis and decides cellular biology and behavior [19]. Many biological processes of mRNA in cancers, such as mRNA splicing, methylation, and interfering, can modulate the mRNA level and alter the cancer property; thereby, mRNA modulation has been a therapeutic target since early times. The levels of mRNAs in cancers, including gliomas, represent the expression of genes that are connected to the prognosis [11]. For glioma prognostic models, the largest proportion of models (78 models) are constructed based on mRNA sequencing data (Figure 2), of which 18 models are in the high-quality group.

3.1.1. LGGs. Low-grade gliomas (LGGs) refer to WHO I, II gliomas [9]. Twenty-one mRNA models for LGGs have been reported. Among them, ten are in the high-quality group and nine are in the medium-quality group.

In the high-quality group, nine [20-28] and one [29] confer high and acceptable accuracy, respectively. Common advantages and additional luminous points in the high-quality group are shown. The corresponding AUC for multiple outcome events of 1,3 , and 5 years' survival in the 4 models [22-25] functions more powerful than many other models that predict merely OS. Two models were validated and assessed highly accurately both internally and externally [21, 22]. Su's model [25] is highly accurate, except when it predicted a 5-year survival training set (AUC was 0.711). For Zeng's model [24], despite its lower AUC for the external validation set, the nomogram performed better via c-index and calibration curve.

For the medium-quality group, five models exhibit high accuracy [30-34], three are acceptable [35-37], and one is lowly accurate [38]. Song et al. constructed a 21- [33] and a 20gene model [34] both internally and externally, and the former was validated via four independent datasets. But the many numbers of predictors should be further streamlined. Hsu et al. [31] and Cheng et al. [30] values for AUC are extremely high (above 0.98 and 0.93 , respectively). For deficiencies in this group, the seven models [30-32, 35-38] lack performance estimation in external or internal validation sets, and $\mathrm{Ni}$ et al. did not validate their 25-gene model [37]. 


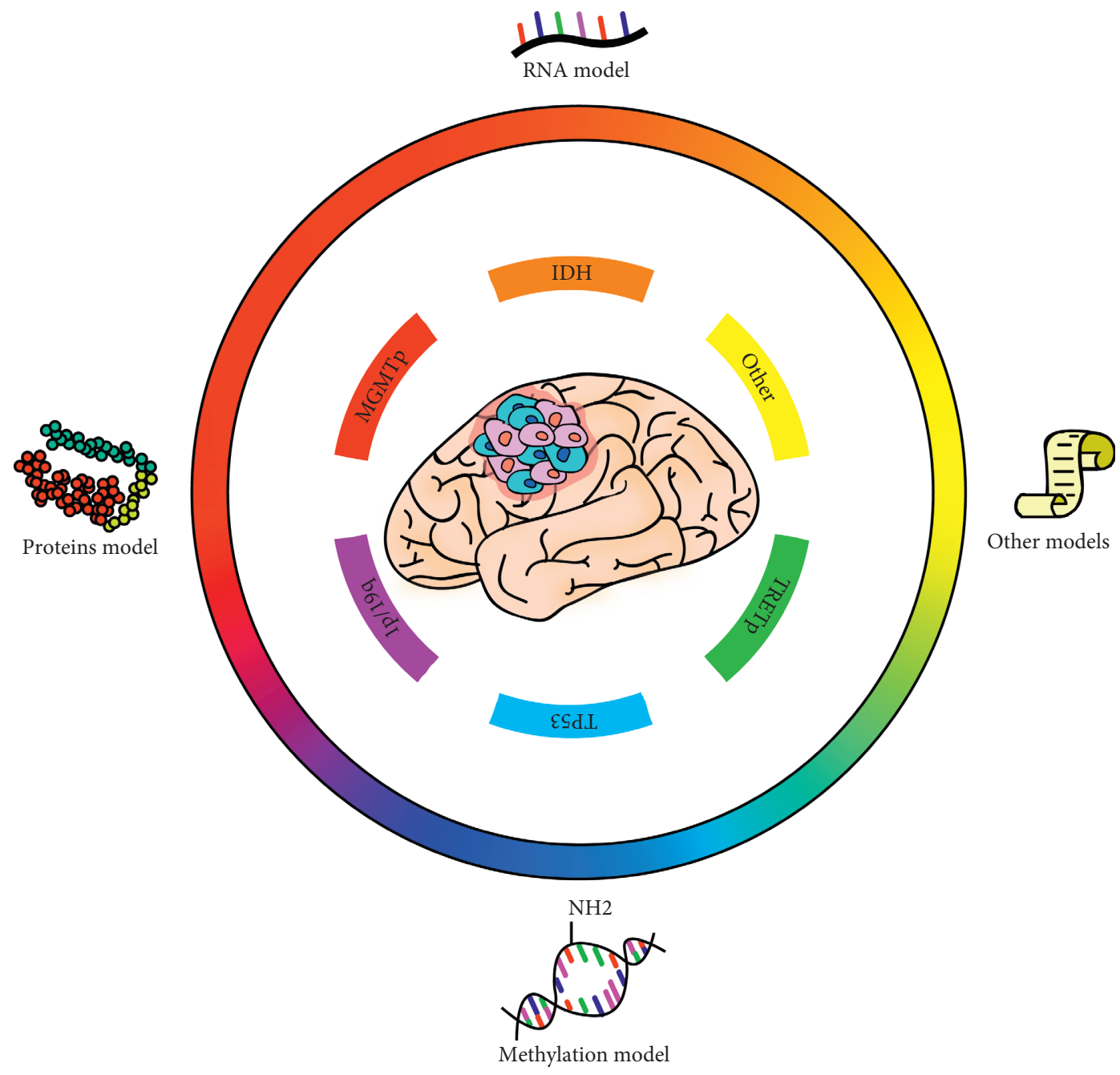

FIGURE 1: Glioma markers and genetic prediction models can stratify patient risks precisely and improve prognostic management. Glioma patient prognosis can be stratified and precisely predicted via prognostic markers and markers-based prediction models and, therefore, improved by adopting corresponding treatment strategies. Markers with prognostic values include IDH, MGMTp, 1p19q, TP53, TERTp, and many other molecules. Prediction models are based on RNA, protein, methylation, and other types of signatures. IDH, isocitrate dehydrogenase; MGMTp, O-6-methylguanine-DNA methyltransferase promoter; TP53, tumor protein p53; TERTp, telomerase reverse transcriptase promoter.

3.1.2. HGGs. High-grade gliomas (HGGs) comprise WHO III and Glioblastoma (GBM). A total of 44 HGGs models have been constructed but show a few satisfactory studies. The high-quality and medium-quality groups contain 7 and 11 models, respectively.

The high-quality models were designed for GBM [39-45]. 4-gene [44] and 3-gene [43] models associated with autophagy were both validated via two independent datasets. The two risk scores' predictive discrimination varies among survival rates in different years and datasets, while their nomograms stabilized the accuracy above 0.72 , which integrated risk scores with other common factors. Nomograms' superior predictive accuracy to risk scores can also be observed in a study by Wang, the nomogram is highly accurate (from 0.77 to 0.85 ) compared with the AUC of risk score (from 0.67 to 0.79 ). Of note, nomogram performance decreased when estimated by c-index [39]. However, in a study by Zhu, the risk score outperformed the nomogram.
AUC of risk score is 0.781 and 0.771 for 2 and 3 years of survival in the discovery cohort [41], while the c-indices of nomogram are also less than 0.70 [41]. Nomograms benefit from combining risk scores with other predictors, but whether their accuracy increases depends on the factors' quality. Moreover, the calibration curves were plotted and verified the reliability in the four studies [39, 41, 43,44]. Additionally, 2329 samples from multiple cohorts in Zhu's study are the largest sample size in the currently existing models, improving its repeatability and transportability [41].

The medium-quality group is better since eight models are of high accuracy [46-53]. The AUC values in models developed by Zhang et al. [47], Hou et al. [52], and Chai et al. [48] are 0.93, 0.95, and 0.93, respectively. Two models achieved acceptable accuracy $[54,55]$, whereby Cheng's final model attained 0.81 when absorbing clinical features and $1 \mathrm{p} /$ $19 q$ status [55]. One model is lowly accurate [56]. This group is limited by a lack of performance estimation for the 


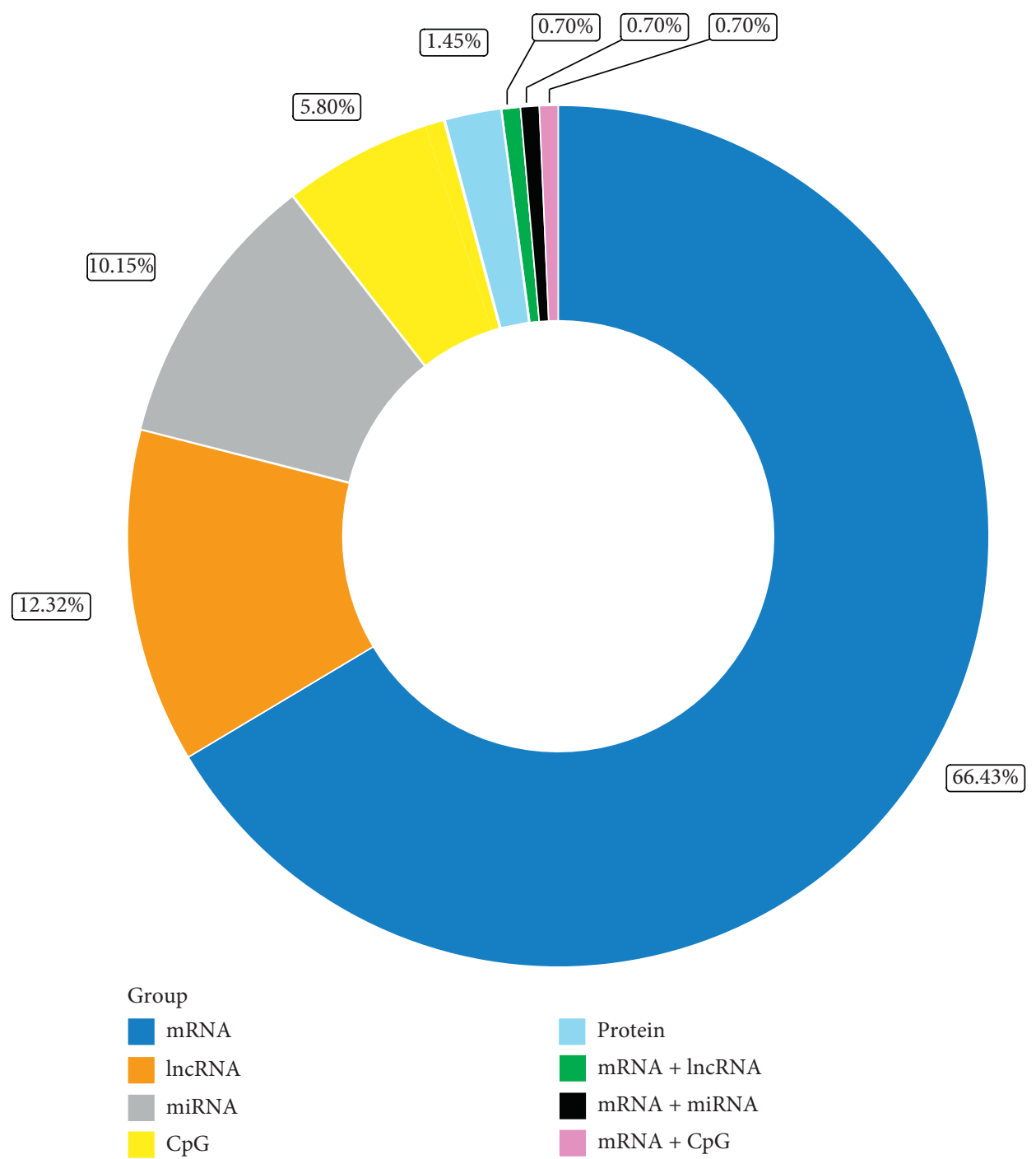

Figure 2: Proportion of published models based on signatures of different molecular types. The largest proportion of 94 mRNA models achieved $68 \%$ of all the 138 genetic prognostic models, followed by 17 lncRNA and 14 miRNA models, accounting for $12 \%$ and $10 \%$, respectively. mRNA, message RNA; lncRNA, long noncoding RNA; miRNA, microRNA.

validation set except for Hou's model, but the weakness of Hou's model is the many predictors (14 genes) [52].

3.1.3. Other Classified Gliomas Clusters. Three models and ten models are grouped into the high-quality and mediumquality groups from the total 28 models reviewed, respectively. The three high-quality models are highly accurate [57-59]. Particularly, Wang's model for diffuse glioma exhibits excellent discrimination with AUC from 0.874 to 0.950 [58]. But it was only internally validated.

Nine medium-quality models show high accuracy [60-68], whereby three models for diffuse glioma estimated accuracy level in training and validation sets despite the large size of predictors $[64,66,67]$. Notably, Sun's model has high accuracy when compared with all the signatures randomly derived from the screen method and outperformed the other three signatures in predicting drug sensitivity [66]. Additionally, it achieved higher accuracy when combined with age, grade, and another signature in the validation set for 3 and 5 years' survival [66]. Other 3 studies (two for all diffuse glioma $[60,65]$, and one for $1 \mathrm{p} / 19 \mathrm{q}$ codeletion diffuse glioma) [62] together with a low-accuracy model for all glioma [69] in the medium-quality group are characterized by missing accuracy estimation in the validation set and excessive predictors.

Conclusively, most studies were designed for diffuse gliomas, and our criteria characterized 3 high-quality models. Besides, many models show high predictive accuracy when subjected to training. However, the absence of accuracy of validation sets failed to affirm the obtained discrimination results.

3.2. LncRNAs. Long noncoding RNAs (lncRNAs) are transcripts more than $200 \mathrm{nt}$ in length. LncRNAs lack significant protein-coding capacity, but their regulatory functions are widely engaged from gene expression to 


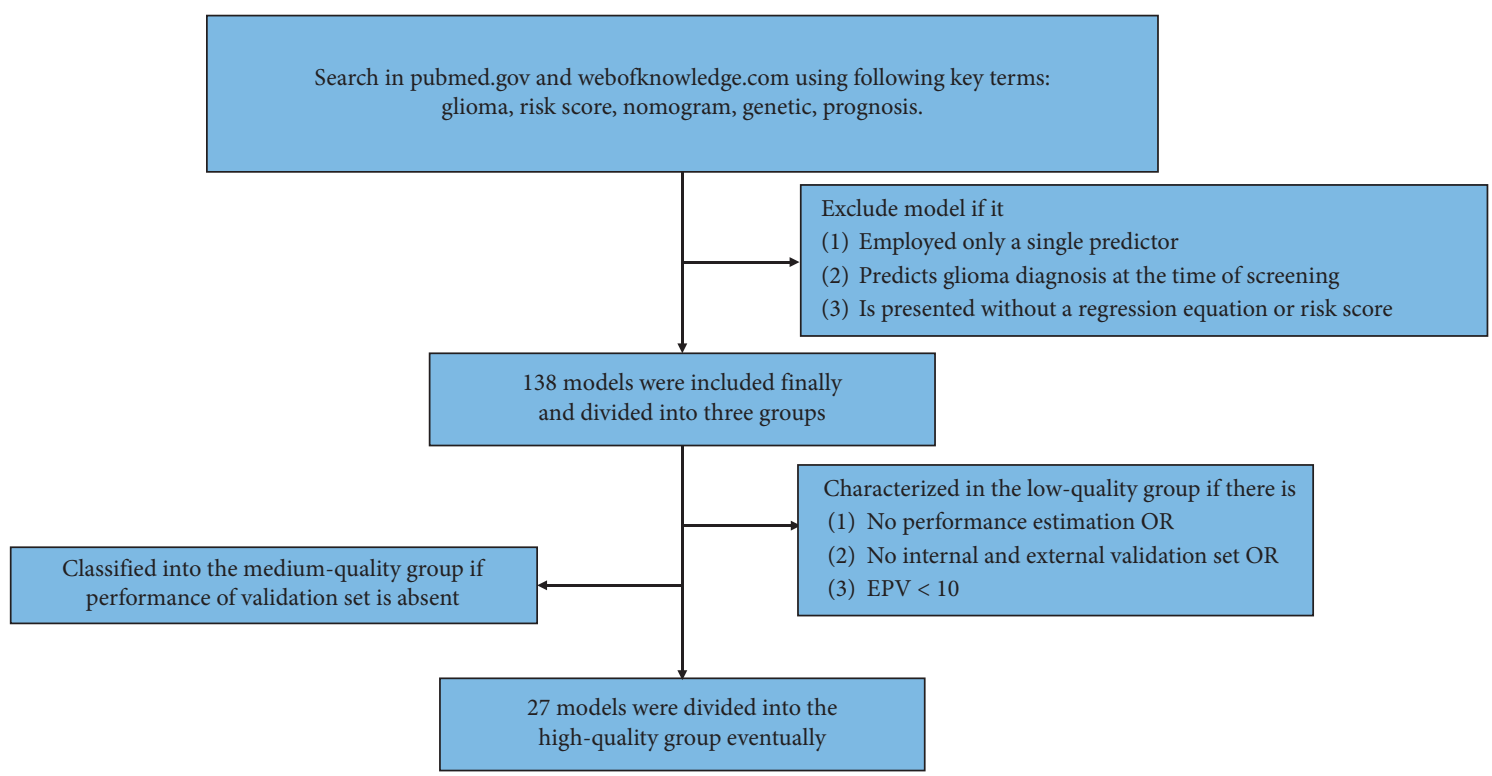

Figure 3: Procedure to screen and classify the genetic prediction models of glioma. The exclusion criteria and grouping rules are proposed firstly in this review, and details are described in Section 2. EPV, events per variable.

TABLE 1: Criteria for model quality estimation.

\begin{tabular}{|c|c|}
\hline Group & Items \\
\hline High-quality group & $\begin{array}{l}\qquad \mathrm{EPV} \geq 10 \text { AND } \\
\text { Variable in final model }<10 \text { AND AUC/c-index estimated for both training and external or internal validation sets }\end{array}$ \\
\hline $\begin{array}{l}\text { Medium-quality } \\
\text { group }\end{array}$ & $\begin{array}{c}\text { EPV } \geq 10 \text { AND } \\
\text { Presence of external or internal validation set but only training set is estimated by receiver operating characteristic } \\
\text { curve/c-index }\end{array}$ \\
\hline Low-quality group & $\begin{array}{c}\text { No performance estimation OR } \\
\text { No internal and external validation set OR EPV }<10\end{array}$ \\
\hline
\end{tabular}

EPV, event per variable.

protein translation. In gliomas, the lncRNAs function in stemness, drug resistance, blood-tumor barrier permeability, angiogenesis, and motility cancer phenotypes [70]. lncRNA is the second major hotspot in model research, after mRNA (Figure 2).

Seventeen lncRNA-signature models on different gliomas and one model on diffuse intrinsic pontine glioma have been reported. Three are high-quality models, and five are medium-quality models.

All three high-quality models exhibit high accuracy [71-73]. Except for the slightly decreased AUC values (0.722) when submitted to external validation based on Chen's study [73], the other two models show similar high accuracy in training, internal validation, and the entire set with AUC from 0.84 to 0.91 [71, 72].

The highest AUC value in the medium-quality group was obtained in Wang's model (0.942) for anaplastic glioma [74]. This is followed by Kiran's UVA8 model (8-lncRNA signature) [75] acceptably test for 5-year survival. The AUC values for the other two models were 0.68 and 0.70 , respectively $[76,77]$. In the five medium-quality models, they were externally validated, but three lacked internal validation [74, 77, 78]. Moreover, Kiran's study reported the UVA8 model and compared it with other predictors and models [75]. The UVA8 accuracy [75] is higher than other clinical features or IDH status. It outperformed the 5 published signatures in the training dataset by c-indices [75]. While the 6 models were designed for a diverse class of gliomas and different prognostic events and to validate various datasets, this positive result in Kiran's study was inevitably questionable due to incomparability.

Internal validation is absent in the 9 low-quality models (Table S1), which is vital to address the stability in selecting predictors and the quality of predictions before clinical application [79]. Cross-validation or bootstrapping methods should be employed to achieve complete internal validation.

3.3. miRNAs. MicroRNAs (miRNAs) are a class of noncoding RNA that binds to complementary target mRNAs. This results in mRNA translational inhibition or degradation. In gliomas, miRNAs are involved in various tumorassociated activities, including immune response, hypoxia, tumor plasticity, and resistance to therapy through multigene targets [80], indicating miRNA-based models as a promising strategy for glioma prognosis.

Fourteen studies on miRNA signatures, one on LGG [81], and others on HGG were reported. Three models were 
characterized in the high-quality group [81-83], whereas the other models were classified in the low-quality group.

The three high-quality models have respective advantages. The 5-miRNA model by Cheng et al. [83] adopted complete internal and external validations, and the AUC values increased from 0.649 and 0.756 to 0.847 and 0.909 after integrating age and chemotherapy, respectively, although only 19 samples were submitted to external validation. Chen's model [82] was internally and externally validated and achieved similar AUC for disease-free survival and OS in three datasets. Besides, Qian's study [81] established a nomogram that predicted a 1-, 2-, 3-, and 5-year survival rate, which is informative for prognostic management. However, its drawbacks include the absence of internal validation and low accuracy in the training set (cindex $=0.68$ ).

\section{Methylation Models}

Cytosine-phosphate-guanine $(\mathrm{CpG})$ islands are a cluster of $\mathrm{CpG}$ sites located at or near the transcription start regions of genes and gene promoters. CpG island methylation is the most common epigenetic type of cancer. The $\mathrm{CpG}$ island methylator phenotype that comprises several CpG islands is an interesting topic in cancer epigenetics [84]. The glioma $\mathrm{CpG}$ island methylator phenotype is associated with gliomas tumorigenesis and is an independent biomarker stratifying gliomas into epigenetic subtypes [85]. Currently, 8 models have been reported and none of them is a high-quality model; 3 of the models are classified into the mediumquality group [86-88].

The two medium-quality models have acceptable discriminations from 0.71 to 0.77 but were not internally validated $[86,87]$. Yin's $6-\mathrm{CpG}$ risk score [86] achieved a higher AUC value (0.734) for patients receiving all treatment integrated with the $\mathrm{CpG}$ island methylator phenotype. Moreover, higher AUC (0.771) was achieved with the MGMT status combination for those receiving radiation therapy/ temozolomide. Besides, the prediction accuracy rate of the $6-\mathrm{CpG}$ signature $(87 \%)$ was validated via the support vector machines model.

\section{Other Multimolecular Models}

Two protein-signature models based on reverse phase protein array were constructed. Stetson's 13-protein risk model [89] applied c-index to estimate the model's accuracy in both training and validating sets for GBM (0.63 and 0.60, respectively) and $I D H$-wildtype LGG (0.82 and 0.70 , respectively), but the shortage is the low EPV (less than 10). Patil and Mahalingam developed another 4-protein model, but without external validation and performance estimation [90]. Both two models are of low quality.

Three mixed models of different classes of molecular signatures were presented for GBMs prognosis. The mixed model for mRNA and lncRNA is of high quality [91], and the other two are medium-quality models $[92,93]$. The three models were fully validated both internally and externally. They were estimated using receiver operating characteristic curve or c-index; however, the validation set lacked estimation, and there was a low c-index value (0.68) in the training set from Etcheverry's study [92].

\section{Biofunction and Clinical Significance of Frequently Reported Molecules}

Molecular signatures reviewed consist of mutated genes, noncoding RNAs, and proteins. Currently, the star markers including IDH, MGMT, 1p/19q, H3K27M, TERT, and ATRX are known to exhibit significant prognostic value. The $I D H$ mutant with $1 \mathrm{p} / 19 \mathrm{q}$ codeletion and MGMT promoter methylation are favorable prognostic factors. The $H 3 \mathrm{~K} 27 \mathrm{M}$ mutant and ATRX alteration are associated with higher risk whereas TERT has a dichotomous prognostic effect [94]. Besides, other molecular signatures collected from 138 published models were reported to contribute to prognostic risk estimation. The prognostic value for most molecular biomarkers has not been validated. Therefore, we analyzed 138 models to select the most overlapping biomarkers (Table 2) with known evidence from researches to determine their biofunctions and potential prognostic values in gliomas. The predictors that presented repeatedly more than twice in 138 model studies are listed in Table 2. Predictors that overlapped less than three times were not reviewed.

6.1. IGFBP2. IGFBP2 regulates insulin-like growth factors' distribution and biofunction by interacting with the insulinlike growth factor system. High aberrant IGFBP2 expression was detected in HGG [95]. This played critical roles in glioma progression and was correlated with poor prognosis [95]. Besides, IGFBP2 downregulation was reported specifically in IDH-mutant gliomas [96]. Besides, EGFR (epidermal growth factor receptor) and integrin $\beta$ can integrate with IGFBP2 to promote tumor progression [95]. The IGFBP2 gene, therefore, presents prognostic value and functions as a potential immunotherapeutic target for GBM in the future clinical trials $[95,96]$.

6.2. HDAC Families and CD44. Histone deacetylase (HDAC) is a vast family of enzymes that mainly exert a repressive influence on transcription [97]. It blocks gene transcription by inhibiting histone acetylation and compacts the DNA/histone complex. In gliomas, $H D A C$ functions to bridge the xCT-CD44 complex with malignant glioma cells and various tumor zones [98]. Currently, HDAC inhibitors exhibit unfavorable therapeutic efficacy in glioma patients. Researchers have reported a more beneficial strategy that adopted HDAC inhibitors in combined therapy [99]. Moreover, many clinical trials (Table 3 ) are ongoing to test their application prospects in treating diffuse intrinsic pontine glioma. and HGG. Currently, HDAC inhibitors are characterized by HDACs risk factors; however, their predictive ability has not been verified. In-depth explorations on their therapeutic efficacy and prognostic value are required. 
TABLE 2: Highly overlapping genes and miRNAs.

\begin{tabular}{lccc}
\hline $\begin{array}{l}\text { Overlapping } \\
\text { times }\end{array}$ & $\begin{array}{c}\text { Molecule } \\
\text { numbers }\end{array}$ & Genes & miRNAs \\
\hline 6 & 1 & IGFBP2 & - \\
5 & 3 & HDAC, STAT1 & miR-222 \\
4 & 6 & GPNMB, VEGFA, EFEMBP2, ISG20, FZD7, EGFR, & - \\
3 & 23 & KI67, BUB1, LAMB1, MAP2K3, KCNB1, KCNJ10, IRF1, ASF1A, SOCS3, & miR-148a, miR-15b, \\
& & KCNR-145, miR-20a & \\
\hline
\end{tabular}

TABLE 3: Completed and active trials primarily completed in 5 years of HDAC, VEGF, and EGFR related therapy.

\begin{tabular}{|c|c|c|c|c|c|}
\hline Gene & Agent name & Glioma type & Clinical trials & Development phase & Status \\
\hline \multirow{5}{*}{ HDAC } & \multirow{4}{*}{ Vorinostat } & DIPG & NCT01189266 & Phase I/II & Active \\
\hline & & DIPG & NCT02420613 & Phase I & Active \\
\hline & & HGG & NCT01236560 & Phase II/III & Completed \\
\hline & & HGG, recurrent GBM & NCT01266031 & Phase I/II & Completed \\
\hline & Belinostat & GBM & NCT02137759 & Phase II & Active \\
\hline \multirow{10}{*}{ VEGF } & \multirow{10}{*}{ Bevacizumab } & Anaplastic glioma, recurrent GBM & NCT01836536 & Unknown & Completed \\
\hline & & Recurrent ependymoma, WHO II, III glioma & NCT00381797 & Phase II & Completed \\
\hline & & GBM & NCT01091792 & Early phase I & Completed \\
\hline & & HGG, recurrent GBM & NCT01266031 & Phase I/ II & Completed \\
\hline & & HGG & NCT01236560 & Phase II/III & Completed \\
\hline & & Recurrent GBM & NCT01648348 & Phase I/II & Completed \\
\hline & & GBM & NCT01498328 & Phase II & Completed \\
\hline & & GBM, oligodendroglioma & NCT01609790 & Phase II & Active \\
\hline & & Recurrent GBM & NCT02142803 & Phase I & Active \\
\hline & & Recurrent GBM & NCT02974621 & Phase II & Active \\
\hline \multirow{16}{*}{ EGFR } & Erlotinib & HGG & NCT01257594 & Phase I & Completed \\
\hline & Lapatinib & Recurrent HGG & NCT02101905 & Phase I & Active \\
\hline & Unknown $^{\dagger}$ & GBM & NCT01454596 & Phase I/II & Completed \\
\hline & AMG 595 & Recurrent GBM, AA & NCT01475006 & Phase I & Completed \\
\hline & Sym004 & Recurrent GBM & NCT02540161 & Phase II & Completed \\
\hline & \multirow{4}{*}{ Depatux-M } & GBM & NCT02343406 & Phase II & Completed \\
\hline & & GBM & NCT02573324 & Phase II/III & Active \\
\hline & & GBM & NCT03419403 & Phase III & Terminated \\
\hline & & GBM & NCT01800695 & Phase I & Completed \\
\hline & \multirow{2}{*}{ Dacomitinib } & Recurrent GBM & NCT01520870 & Phase II & Completed \\
\hline & & GBM & NCT01112527 & Phase II & Completed \\
\hline & Tesevatinib & GBM & NCT02844439 & Phase II & Completed \\
\hline & Afatinib & GBM & NCT00977431 & Phase I & Completed \\
\hline & Cetuximab & GBM, anaplastic astrocytoma & NCT01238237 & Phase I & Completed \\
\hline & \multirow{2}{*}{ Rindopepimut } & GBM & NCT01498328 & Phase II & Completed \\
\hline & & GBM & NCT01480479 & Phase III & Completed \\
\hline
\end{tabular}

DIPG, diffuse intrinsic pontine glioma; HGG, high-grade glioma; GBM, glioblastoma; AA, anaplastic astrocytoma. ${ }^{\dagger}$ Epidermal growth factor receptor (EGFRv) III; chimeric antigen receptor (CAR); transduced peripheral blood lymphocytes (PBL).

CD44 was identified in GBM and from brain metastases [100]. It is a biomarker of the mesenchymal GBM subtype with the most aggressive growth patterns [101]. Besides, GBM progression was inhibited by inhibiting CD44 expression. This indicates the roles of CD44 in the tumor process [102].

6.3. $M D K . M D K$ is a heparin-binding growth factor encoding gene extensively studied for its multiple functions in various tissues. $M D K$ contributes to numerous tumorrelated activities in glioma, and its overexpression is associated with poor prognosis [103]. However, no clinical trial has been performed to explain its therapeutic potential.
6.4. GPNMB. GPNMB encodes the type 1 transmembrane protein expressed mainly on the surface of cancer cells [104]. $G P N M B$ promotes tumor progression through immunemicroenvironment plasticity. It also enhances $\mathrm{Wnt} / \beta$-catenin signaling pathway activation and interaction with $\mathrm{Na}^{+} / \mathrm{K}^{+}$ATPase subunits [105-107]. Also, abnormally high GPNMB expression has been reported to be associated with unfavorable survival outcomes in GBM [108]. These mechanisms justify that GPNMB is a risk factor for glioma patients.

6.5. EGFR. It has been reported that EGFR signaling pathways are activated in the majority of GBM cells [109]. EGFR gene aberration contains rearrangement, 
amplification, and mutation. The EGFR variant III (EGFRvIII) is a common mutation product in GBM [110]. The EGFRvIII contributes to many tumor biological features [111], indicating a poor outcome. Besides, wild-type EGFR associated with tumor cell invasion and angiogenesis has been demonstrated in several in vivo and in vitro experiments [112]. Thus, EGFR and EGFRvIII have been identified as popular therapeutic targets for treating malignant glioma patients. However, current treatments that target EGFR have failed in clinical trials including the small molecule drugs and biologic antibodies [112]. While some trials are still ongoing (Table 3 ).

6.6. VEGFA. VEGFA, also known as VEGF, is a growth factor that promotes tumor angiogenesis and vascular permeability and regulates immune cell and fibroblastoma and microenvironment formation [113]. In glioma, VEGF acts as a regulatory growth factor secreted by glioma stem cells to promote the tumor vasculature [114]. Anti-VEGF-A antibody has been applied as a novel antiangiogenic therapy for malignant gliomas, as with bevacizumab [114]. However, it has not achieved considerable progression in treating gliomas. A report by Eskilsson et al. [112] indicates that causes of failure may be attributed to enhanced invasive properties of tumor cells by different mechanisms, for example, RTK signaling. Many trials using bevacizumab with other strategies, for example, EGFR inhibitors, showed improved efficacy (Table 3); some trials are underway. Since several VEGFA studies on gliomas exist, it is expected that it will work as an accurate and effective predictor for glioma prognosis. This is despite its current poor performance in antiangiogenesis therapy.

6.7. $m i R-221 / m i R-222 . m i R-221 / m i R-222$ are two closely related miRNAs located in the genome region of the $\mathrm{X}$ chromosome. They have a similar sequence, structure, and biofunction and upregulate expression in various human cancers including glioma. Knocking down miR-221/miR222 expression blocks cell cycle transition, suppresses tumor cell growth, and increases sensitivity to radiotherapy [115]. Also, miR-221/miR-222 is associated with tumor cell apoptosis by targeting the apoptosis-related gene [116]. Recent studies revealed its connection with glioma histology and patient prognosis [117]. This showed that either miR-221 or miR-222 expression was associated with a higher WHO grade thereby indicating a poor prognosis.

6.8. Other Significant Predictors. Apart from the abovediscussed vital parameters, other factors play significant roles in glioma malignant properties and prognosis assessment. These predictors include MYC, KCNJ10, CHI3L1, STAT1, and FZD7. MYC amplification has been identified in many classes of cancers. It acted as a qualified prognostic prediction biomarker [118]. KCNJ10 encodes inwardly rectifying potassium channel Kir4.1 protein that is expressed exclusively in central nervous system glial cells. This establishes a hyperpolarized resting membrane potential and prevents glioma cell proliferation [119]. CHI3L1 overexpression is associated with poor survival [120] causing tumor invasion, migration, angiogenesis, and resistance to temozolomide therapy [121]. The aberrant STAT1 activation may cause oncogenesis [122]. Limited information on FZD7 activity in gliomas exists; however, the other four predictors have attracted extensive research.

\section{Discussion}

For model variables, previous studies focused on pathological, anatomic, and clinical predictors, but recently genetic data were widely recommended for enhancing predictive ability $[123,124]$. Formulated genetic predictors harbor an advantage in calculating absolute risks based on marker expression levels via sequencing analysis and coefficients, thus providing stronger information compared with relative risk tools. However, they are still not recommended for clinical application, due to limitations, such as the lack of gold markers, difficulties of data collection, the complexity of analysis, and low adherence to complete and transparent reporting [125]. Our review confirmed a similar problem in gliomas. We found that only 27 models (20\%) are classified into the high-quality group (Table 4$), 31$ models (22\%) are in the medium-quality group, and 83 models $(58 \%)$ are classified in the low-quality groups; none can be clinically applied according to our criteria, which was urgently required to address the issue that no method for assessing the model quality exists currently [125].

Problems in models consist of methodological deficiencies and clinical confirmation absence. The former was mainly attributed to the low adherence to guidelines like the TRIPOD statement [13], leading to series of deficiencies like the lack of performance assessment, validation that was also observed in other cancers [123, 126, 127]. A good example of a prostate cancer model was constructed in transparent and clear details [128], and its constructing details were listed in a table, facilitating the check of model reliability for users and avoiding methodological negligence during the establishment process. And for validating, age-related risk score [129] was analyzed through 2953 cases from 10 datasets; the large sample size and high AUCs verified its robustness. For improving the model methodology, complete and transparent reporting should be strictly ensured. Besides, Zhang et al. highlighted that reference genome and annotation updates cause inconsistent gene expression levels, leading to discordant individual risk grouping. Using up-to-date reference genome, stable gene in each annotation release (with consistent length and overlap), and gene pairs is helpful [18]. For clinical application, while decision curve analysis [130] compares the net benefit of the models with traditional approaches, the best methods for testing clinical significance are prospect clinical trials $[123,125,126]$. Health economic impact evaluations should also be considered [131], but we found that no study has reported the cost of predictor detection. Economic cost and effectiveness of models were critical for the medical decision-maker, like cost-effectiveness ratio; they also contributed to the optimization of risk thresholds. However, 
TABLE 4: Characteristics of 27 high-quality models.

\begin{tabular}{|c|c|c|c|c|c|c|c|}
\hline Authors & Glioma type & Parameters & Sample size ${ }^{a}$ & Validation $^{\mathrm{b}}$ & $\begin{array}{l}\text { Performance } \\
\text { estimation }^{c}\end{array}$ & Accuracy $^{\mathrm{d}}$ & Reference \\
\hline Liu et al. & LGG & 2 mRNAs & $115 /-/ 41$ & E & $\mathrm{T}, \mathrm{E}$ & Low $(0.735)$ & [29] \\
\hline Xiao et al. & LGG & 3 mRNAs & $456 /-/ 159$ & $\mathrm{I}, \mathrm{E}$ & $\mathrm{T}, \mathrm{E}$ & $\operatorname{High}(0.908,0.878,0.827)^{\mathrm{e}}$ & [23] \\
\hline Chen et al. & LGG & 3 mRNAs & $164 /-/ 599$ & $\mathrm{E}$ & $\mathrm{T}, \mathrm{E}$ & High $(0.869)$ & {$[28]$} \\
\hline Zeng et al. & LGG & 4 mRNAs & $172 /-/ 451$ & $\mathrm{E}$ & $\mathrm{T}, \mathrm{E}$ & $\operatorname{High}(0.845,0.890,0.912)^{\mathrm{e}}$ & {$[24]$} \\
\hline $\begin{array}{l}\text { Zhang } \\
\text { et al. }\end{array}$ & LGG & 4 mRNAs & $534 /-/ 325$ & $\mathrm{E}$ & $\mathrm{T}, \mathrm{E}$ & High $(0.858,0.853,0.837)^{\mathrm{e}}$ & {$[27]$} \\
\hline Liu et al. & LGG & 5 mRNAs & $524 /-/ 169$ & I, E & $\mathrm{T}, \mathrm{E}$ & High (0.887) & {$[26]$} \\
\hline Li et al. & LGG & 4 mRNAs +6 mRNAs & $516 /-/ 426$ & $\mathrm{E}$ & $\mathrm{T}, \mathrm{E}$ & High (0.84) & {$[20]$} \\
\hline $\begin{array}{l}\text { Zhang } \\
\text { et al. }\end{array}$ & LGG & 6 mRNAs & $304 / 128 / 353$ & $\mathrm{I}, \mathrm{E}$ & $\mathrm{T}, \mathrm{I}, \mathrm{E}$ & High (0.914) & {$[21]$} \\
\hline $\begin{array}{l}\text { Zhang } \\
\text { et al. }\end{array}$ & LGG & 7 mRNAs & $297 / 124 / 353$ & $\mathrm{I}, \mathrm{E}$ & $\mathrm{T}, \mathrm{I}, \mathrm{E}$ & $\begin{array}{l}\text { High and acceptable }(0.899, \\
0.875,0.778)^{\mathrm{e}}\end{array}$ & {$[22]$} \\
\hline Su et al. & LGG & 8 mRNAs & $511 /-/ 172$ & $\mathrm{E}$ & $\mathrm{T}, \mathrm{E}$ & $\begin{array}{l}\text { High and acceptable }(0.882, \\
0.831,0.711)^{\mathrm{e}}\end{array}$ & {$[25]$} \\
\hline Qian et al. & LGG & 4 miRNAs & $100 /-/ 420$ & $\mathrm{E}$ & $\mathrm{T}, \mathrm{E}$ & Low $(0.680)$ & {$[81]$} \\
\hline $\begin{array}{l}\text { Wang } \\
\text { et al. }\end{array}$ & GBM & 3 mRNAs & $155 /-/ 216$ & $\mathrm{E}$ & $\mathrm{T}, \mathrm{E}$ & High $(0.832)^{\mathrm{f}}$ & {$[43]$} \\
\hline $\begin{array}{l}\text { Wang } \\
\text { et al. }\end{array}$ & GBM & 4 mRNAs & $241 / 160 /-$ & I & $\mathrm{T}, \mathrm{I}$ & $\begin{array}{l}\text { High and acceptable }(0.756 \text {, } \\
0.821,0.885)^{\text {ef }}\end{array}$ & {$[44]$} \\
\hline $\begin{array}{l}\text { Wang } \\
\text { et al. }\end{array}$ & GBM & 5 mRNAs & $364 / 155 /-$ & I & $\mathrm{T}, \mathrm{I}$ & Acceptable $(0.729)^{\mathrm{f}}$ & {$[39]$} \\
\hline Zhao et al. & GBM & 6 mRNAs & $152 /-/ 138$ & $\mathrm{E}$ & $\mathrm{T}, \mathrm{E}$ & High (0.908) & {$[40]$} \\
\hline Zhu et al. & GBM & 5 mRNAs & $\begin{array}{c}306 / 325 / \\
1957\end{array}$ & $\mathrm{I}, \mathrm{E}$ & $\mathrm{T}, \mathrm{I}, \mathrm{E}$ & Acceptable $(0.704)^{\mathrm{f}}$ & {$[41]$} \\
\hline Zuo et al. & GBM & 6 mRNAs & $137 /-/ 158$ & $\mathrm{E}$ & $\mathrm{T}, \mathrm{E}$ & $\begin{array}{c}\text { Acceptable (1-year } 0.699,2 \text {-year } \\
0.779)\end{array}$ & {$[42]$} \\
\hline Gong et al. & GBM & 8 mRNAs & $151 /-/ 138$ & $\mathrm{E}$ & $\mathrm{T}, \mathrm{E}$ & High (0.977) & {$[45]$} \\
\hline Zhou et al. & GBM & 6 lncRNAs & $200 / 219 /-$ & I & $\mathrm{T}, \mathrm{I}$ & High (0.902) & {$[72]$} \\
\hline Chen et al. & GBM & 4 lncRNAs & $240 /-/ 80$ & $\mathrm{E}$ & $\mathrm{T}, \mathrm{E}$ & High (0.843) & {$[73]$} \\
\hline Chen et al. & GBM & 7 miRNAs & $89 / 102 / 109$ & I, E & $\mathrm{T}, \mathrm{I}, \mathrm{E}$ & Low $(0.690)$ & [82] \\
\hline $\begin{array}{l}\text { Cheng } \\
\text { et al. }\end{array}$ & GBM & 5 miRNAs & $75 / 75 / 19$ & $\mathrm{E}$ & $\mathrm{T}, \mathrm{E}$ & High $(0.847)^{\mathrm{f}}$ & [83] \\
\hline Gao et al. & GBM & $\begin{array}{l}6 \text { mRNAs }+5 \\
\operatorname{lncRNAs}\end{array}$ & $76 / 77 / 80$ & I, E & $\mathrm{T}, \mathrm{E}$ & Acceptable (0.780) & {$[91]$} \\
\hline Peng et al. & $\begin{array}{l}\text { Diffuse } \\
\text { glioma }\end{array}$ & 5 mRNAs & $641 /-/ 319$ & $\mathrm{E}$ & $\mathrm{T}, \mathrm{E}$ & $\begin{array}{c}\text { High (3-year } 0.895,5 \text {-year } \\
0.864)\end{array}$ & [59] \\
\hline Wu et al. & Glioma & 9 mRNAs & $550 /-/ 309$ & $\mathrm{E}$ & $\mathrm{T}, \mathrm{E}$ & High (0.860) & {$[57]$} \\
\hline $\begin{array}{l}\text { Wang } \\
\text { et al. }\end{array}$ & Glioma & 5 mRNAs & $420 / 178 /-$ & I & $\mathrm{T}, \mathrm{I}$ & High $(0.917,0.950,0.881)^{\mathrm{e}}$ & {$[58]$} \\
\hline $\mathrm{Hu}$ et al. & Glioma & 5 lncRNAs & $70 / 70 /-$ & I & $\mathrm{T}, \mathrm{I}$ & High (0.910) & {$[71]$} \\
\hline
\end{tabular}

${ }^{a}$ Sample size of training set/internal validation set/external validation set. ${ }^{\mathrm{b}} \mathrm{I}$ and $\mathrm{E}$ represent the presence of internal and external validation, respectively. ${ }^{\mathrm{c}} \mathrm{T}$, I, and $\mathrm{E}$ represent the presence of performance estimation in training, internal validation, and external validation sets, respectively. ${ }^{\mathrm{d}}$ Models were classified into high, acceptable, and low accuracy according to AUC or c-index as we present in rules for judging model quality and excision criteria part, and accuracy in only the training set is shown. ${ }^{\mathrm{e}}$ Three AUC values for prediction of 1-, 3-, and 5-year survival, respectively. ${ }^{\mathrm{f}}$ If higher accuracy was achieved by nomogram or integrated model, the highest accuracy result was exhibited instead of the result of the original risk score.

since few studies have conducted these methods, we emphasized increasing awareness of clinical considerations when a high-quality model was established.

Conclusively, we comprehensively reviewed all 138 machine learning genetic models in gliomas and proposed novel criteria that will foster the development or assessment of clinically important models for not only neurosurgeons but also researchers in other cancers. Given the current situation, a lot of effort should be put to standardize the model quality through adherence to complete and transparent reporting and promote model generalization by conducting prospective clinical trials, and economic effectiveness should be the following issue. Despite the various difficulties, future genetic models will lead the prognostic management, and novel gene-pair-based models deserve development.

\section{Abbreviations}

AUC: Area under the curve

EGFR: Epidermal growth factor receptor

EPV: $\quad$ Events per variable

GBM: Glioblastoma multiforme

HGG: High-grade glioma

LGG: $\quad$ Low-grade glioma

lncRNA: Long noncoding RNA 
miRNA: Micro RNA

OS: $\quad$ Overall survival

WHO: World Health Organization.

\section{Data Availability}

No data were used to support this study.

\section{Conflicts of Interest}

The authors declare that there are no conflicts of interest regarding the publication of this article.

\section{Authors' Contributions}

Conception and design were done by Quan Cheng and Zhixiong Liu; administrative support was given by Quan Cheng and Zhixiong Liu; provision of study materials or patients was done by Xisong Liang and Zeyu Wang; collection and assembly of data were carried out by Ziyu Dai and Hao Zhang; data analysis and interpretation were performed by Xisong Liang and Zeyu Wang; manuscript writing was done by all authors (VII). All authors approved the final version of the manuscript.

\section{Acknowledgments}

This work was supported by the National Natural Science Foundation of China (NO. 82073893 to Q. C., NO. 81873635 to Z. L., and NO. 81703622 to Q. C.), the China Postdoctoral Science Foundation (NO. 2018M633002 to Q. C.), the Natural Science Foundation of Hunan Province (NO. 2018JJ3838 to Q. C. and NO. 2018SK2101 to Z. L.), the Hunan Provincial Health and Health Committee Foundation of China (NO. C2019186 to Q. C.), and Xiangya Hospital Central South University Postdoctoral Foundation (to Q. C.).

\section{Supplementary Materials}

Table S1: characteristics of 73 models in the low-quality group. (Supplementary Materials)

\section{References}

[1] C. P. L. Simmons, D. C. McMillan, K. McWilliams et al., "Prognostic tools in patients with advanced cancer: a systematic review," Journal of Pain and Symptom Management, vol. 53, no. 5, pp. 962-970, 2017.

[2] K. Y. Ngiam and I. W. Khor, "Big data and machine learning algorithms for health-care delivery," The Lancet Oncology, vol. 20, no. 5, pp. e262-e273, 2019.

[3] R. Liang, Y. Zhi, G. Zheng, B. Zhang, H. Zhu, and M. Wang, "Analysis of long non-coding RNAs in glioblastoma for prognosis prediction using weighted gene co-expression network analysis, Cox regression, and L1-LASSO penalization," OncoTargets and Therapy, vol. 12, pp. 157-168, 2019.

[4] N. Zhang, Z. Dai, W. Wu et al., "The predictive value of monocytes in immune microenvironment and prognosis of glioma patients based on machine learning," Frontiers in Immunology, vol. 12, Article ID 656541, 2021.
[5] K. G. M. Moons, P. Royston, Y. Vergouwe, D. E. Grobbee, and D. G. Altman, "Prognosis and prognostic research: what, why, and how?” BMJ, vol. 338, p. b375, 2009.

[6] D. A. Grimes, "The nomogram epidemic: resurgence of a medical relic," Annals of Internal Medicine, vol. 149, no. 4, pp. 273-275, 2008.

[7] E. W. Steyerberg and F. E. Harrell Jr., "Prediction models need appropriate internal, internal-external, and external validation," Journal of Clinical Epidemiology, vol. 69, pp. 245-247, 2016.

[8] J. J. Miller, F. Loebel, T. A. Juratli et al., "Accelerated progression of IDH mutant glioma after first recurrence," Neuro-Oncology, vol. 21, no. 5, pp. 669-677, 2019.

[9] D. N. Louis, H. Ohgaki, O. D. Wiestler et al., "The 2007 WHO classification of tumours of the central nervous system," Acta Neuropathologica, vol. 114, no. 2, pp. 97-109, 2007.

[10] D. J. Brat, R. G. Verhaak, K. D. Aldape et al., "Comprehensive, integrative genomic analysis of diffuse lower-grade gliomas," New England Journal of Medicine, vol. 372, no. 26, pp. 2481-2498, 2015.

[11] D. N. Louis, A. Perry, G. Reifenberger et al., "The 2016 world health organization classification of tumors of the central nervous system: a summary," Acta Neuropathologica, vol. 131, no. 6, pp. 803-820, 2016.

[12] A. M. Molinaro, M. R. Wrensch, R. B. Jenkins, and J. E. Eckel-Passow, "Statistical considerations on prognostic models for glioma," Neuro-Oncology, vol. 18, no. 5, pp. 609-623, 2016.

[13] G. S. Collins, J. B. Reitsma, D. G. Altman, and K. G. M. Moons, "Transparent reporting of a multivariable prediction model for individual prognosis or diagnosis (TRIPOD): the TRIPOD statement," European Urology, vol. 67, no. 6, pp. 1142-1151, 2015.

[14] A. C. Alba, T. Agoritsas, M. Walsh et al., "Discrimination and calibration of clinical prediction models," JAMA, vol. 318, no. 14, pp. 1377-1384, 2017.

[15] D. G. Altman, Y. Vergouwe, P. Royston, and K. G. M. Moons, "Prognosis and prognostic research: validating a prognostic model,” BMJ, vol. 338, p. b605, 2009.

[16] B. S. Wessler, L. Lai Yh, W. Kramer et al., "Clinical prediction models for cardiovascular disease," Circulation: Cardiovascular Quality and Outcomes, vol. 8, no. 4, pp. 368-375, 2015.

[17] E. O. Ogundimu, D. G. Altman, and G. S. Collins, "Adequate sample size for developing prediction models is not simply related to events per variable," Journal of Clinical Epidemiology, vol. 76, pp. 175-182, 2016.

[18] Z. Zhang, S. Zhang, X. Li et al., "Reference genome and annotation updates lead to contradictory prognostic predictions in gene expression signatures: a case study of resected stage I lung adenocarcinoma," Briefings in Bioinformatics, vol. 22, no. 3, p. bbaa081, 2020.

[19] F. Crick, "Central dogma of molecular biology," Nature, vol. 227, no. 5258, pp. 561-563, 1970.

[20] G. Li, Y. Jiang, X. Lyu et al., "Gene signatures based on therapy responsiveness provide guidance for combined radiotherapy and chemotherapy for lower grade glioma," Journal of Cellular and Molecular Medicine, vol. 24, no. 8, pp. 4726-4735, 2020.

[21] M. Zhang, X. Wang, X. Chen, Q. Zhang, and J. Hong, "Novel immune-related gene signature for risk stratification and prognosis of survival in lower-grade glioma," Frontiers in Genetics, vol. 11, p. 363, 2020.

[22] M. Zhang, X. Wang, X. Chen, F. Guo, and J. Hong, "Prognostic value of a stemness index-associated signature in 
primary lower-grade glioma," Frontiers in Genetics, vol. 11, p. $441,2020$.

[23] K. Xiao, Q. Liu, G. Peng, J. Su, C.-Y. Qin, and X.-Y. Wang, "Identification and validation of a three-gene signature as a candidate prognostic biomarker for lower grade glioma," PeerJ, vol. 8, Article ID e8312, 2020.

[24] F. Zeng, X. Liu, K. Wang, Z. Zhao, and G. Li, “Transcriptomic profiling identifies a DNA repair-related signature as a novel prognostic marker in lower grade gliomas," Cancer Epidemiology, Biomarkers \& Prevention, vol. 28, no. 12, 2019.

[25] J. Su, W. Long, Q. Ma et al., "Identification of a tumor microenvironment-related eight-gene signature for predicting prognosis in lower-grade gliomas," Frontiers in Genetics, vol. 10, p. 1143, 2019.

[26] B. Liu, J. Liu, K. Liu et al., "A prognostic signature of five pseudogenes for predicting lower-grade gliomas," Biomedicine \& Pharmacotherapy, vol. 117, Article ID 109116, 2019.

[27] C. Zhang, R. Yu, Z. Li et al., "Comprehensive analysis of genes based on chrlp/19q co-deletion reveals a robust 4-gene prognostic signature for lower grade glioma," Cancer Management and Research, vol. 11, pp. 4971-4984, 2019.

[28] B. Chen, T. Liang, P. Yang et al., "Classifying lower grade glioma cases according to whole genome gene expression," Oncotarget, vol. 7, no. 45, pp. 74031-74042, 2016.

[29] Q. Liu, K. Wang, R. Huang et al., "A novel DNA damage response signature of IDH-mutant grade II and grade III astrocytoma at transcriptional level," Journal of Cancer Research and Clinical Oncology, vol. 146, no. 3, pp. 579-591, 2020.

[30] W. Cheng, X. Ren, C. Zhang, J. Cai, S. Han, and A. Wu, "Gene expression profiling stratifies IDH1-mutant glioma with distinct prognoses," Molecular Neurobiology, vol. 54, no. 8, pp. 5996-6005, 2017.

[31] J. B.-K. Hsu, T.-H. Chang, G. A. Lee, T.-Y. Lee, and C.-Y. Chen, "Identification of potential biomarkers related to glioma survival by gene expression profile analysis," BMC Medical Genomics, vol. 11, no. S7, p. 34, 2019.

[32] G. Li, Y. Jiang, X. Lyu et al., "Deconvolution and network analysis of IDH-mutant lower grade glioma predict recurrence and indicate therapeutic targets," Epigenomics, vol. 11, no. 11, pp. 1323-1333, 2019.

[33] L.-R. Song, J.-C. Weng, X.-L. Huo et al., "Identification and validation of a 21-mRNA prognostic signature in diffuse lower-grade gliomas," Journal of Neuro-Oncology, vol. 146, no. 1, pp. 207-217, 2020.

[34] L. R. Song, J. C. Weng, C. B. Li et al., "Prognostic and predictive value of an immune infiltration signature in diffuse lower-grade gliomas," JCI Insight, vol. 5, no. 8, 2020.

[35] W.-J. Zeng, Y.-L. Yang, Z.-Z. Liu et al., "Integrative analysis of DNA methylation and gene expression identify a threegene signature for predicting prognosis in lower-grade gliomas," Cellular Physiology and Biochemistry, vol. 47, no. 1, pp. 428-439, 2018.

[36] R. C. Chai, N. Wang, Y. Z. Chang et al., "Systematically profiling the expression of eIF3 subunits in glioma reveals the expression of eIF3i has prognostic value in IDH-mutant lower grade glioma," Cancer Cell International, vol. 19, p. $155,2019$.

[37] J. Ni, S. Liu, F. Qi et al., "Screening TCGA database for prognostic genes in lower grade glioma microenvironment," Annals of Translational Medicine, vol. 8, no. 5, p. 209, 2020.

[38] W. Wang, J. Li, F. Lin, J. Guo, and J. Zhao, "Expression and prognostic value of mRNAs in lower grade glioma with
MGMT promoter methylated," Journal of Clinical Neuroscience, vol. 75, pp. 45-51, 2020.

[39] Y. Wang, X. Liu, G. Guan, W. Zhao, and M. Zhuang, "A risk classification system with five-gene for survival prediction of glioblastoma patients," Frontiers in Neurology, vol. 10, p. 745, 2019.

[40] J. Zhao, L. Wang, G. Hu, and B. Wei, “A 6-gene risk signature predicts survival of glioblastoma multiforme," Biomed Research International, vol. 2019, Article ID 1649423, , 2019.

[41] C. Zhu, C. Zou, G. Guan et al., "Development and validation of an interferon signature predicting prognosis and treatment response for glioblastoma," Oncoimmunology, vol. 8, no. 9, Article ID e1621677, 2019.

[42] S. Zuo, X. Zhang, and L. Wang, "A RNA sequencing-based six-gene signature for survival prediction in patients with glioblastoma," Scientific Reports, vol. 9, no. 1, p. 2615, 2019.

[43] Z. Wang, L. Gao, X. Guo et al., "Development and validation of a nomogram with an autophagy-related gene signature for predicting survival in patients with glioblastoma," Aging, vol. 11, no. 24, pp. 12246-12269, 2019.

[44] Y. Wang, W. Zhao, Z. Xiao, G. Guan, X. Liu, and M. Zhuang, "A risk signature with four autophagy-related genes for predicting survival of glioblastoma multiforme," Journal of Cellular and Molecular Medicine, vol. 24, no. 7, pp. 38073821, 2020.

[45] Z. Gong, F. Hong, H. Wang, X. Zhang, and J. Chen, "An eight-mRNA signature outperforms the lncRNA-based signature in predicting prognosis of patients with glioblastoma," BMC Medical Genetics, vol. 21, no. 1, p. 56, 2020.

[46] M. De Tayrac, M. Aubry, S. Saïkali et al., "A 4-gene signature associated with clinical outcome in high-grade gliomas," Clinical Cancer Research, vol. 17, no. 2, pp. 317-327, 2011.

[47] C.-B. Zhang, P. Zhu, P. Yang et al., "Identification of high risk anaplastic gliomas by a diagnostic and prognostic signature derived from mRNA expression profiling," Oncotarget, vol. 6, no. 34, pp. 36643-36651, 2015.

[48] R. Chai, K. Zhang, K. Wang et al., "A novel gene signature based on five glioblastoma stem-like cell relevant genes predicts the survival of primary glioblastoma," Journal of Cancer Research and Clinical Oncology, vol. 144, no. 3, pp. 439-447, 2018.

[49] Q. Wang, J. Cai, C. Fang et al., "Mesenchymal glioblastoma constitutes a major ceRNA signature in the TGF- $\beta$ pathway," Theranostics, vol. 8, no. 17, pp. 4733-4749, 2018.

[50] S. Jin, Z. Qian, T. Liang et al., "Identification of a DNA repair-related multigene signature as a novel prognostic predictor of glioblastoma," World Neurosurgery, vol. 117, pp. E34-E41, 2018.

[51] N. Ye, N. Jiang, C. Feng et al., "Combined therapy sensitivity index based on a 13-gene signature predicts prognosis for IDH wild-type and MGMT promoter unmethylated glioblastoma patients," Journal of Cancer, vol. 10, no. 22, pp. 5536-5548, 2019.

[52] Z. Hou, J. Yang, H. Wang, D. Liu, and H. Zhang, “A potential prognostic gene signature for predicting survival for glioblastoma patients," BioMed Research International, vol. 2019, Article ID 9506461, , 2019.

[53] P. Liang, Y. Chai, H. Zhao, and G. Wang, "Predictive analyses of prognostic-related immune genes and immune infiltrates for glioblastoma," Diagnostics (Basel, Switzerland), vol. 10, no. 3, p. 177, 2020.

[54] Z.-S. Bao, C.-B. Zhang, H.-J. Wang et al., "Whole-genome mRNA expression profiling identifies functional and prognostic signatures in patients with mesenchymal glioblastoma 
multiforme," CNS Neuroscience \& Therapeutics, vol. 19, no. 9, pp. 714-720, 2013.

[55] Q. Cheng, C. Huang, H. Cao et al., "A novel prognostic signature of transcription factors for the prediction in patients with GBM," Frontiers in Genetics, vol. 10, p. 906, 2019.

[56] W. Wang, Z. Zhao, F. Wu et al., "Bioinformatic analysis of gene expression and methylation regulation in glioblastoma," Journal of Neuro-Oncology, vol. 136, no. 3, pp. 495-503, 2018.

[57] F. Wu, Z. Zhao, R. C. Chai et al., "Prognostic power of a lipid metabolism gene panel for diffuse gliomas," Journal of Cellular and Molecular Medicine, vol. 23, no. 11, pp. 77417748, 2019.

[58] Y. Wang, X. Liu, G. Guan, Z. Xiao, W. Zhao, and M. Zhuang, "Identification of a five-pseudogene signature for predicting survival and its ceRNA network in glioma," Frontiers in Oncology, vol. 9, p. 1059, 2019.

[59] Z. Peng, Y. Chen, H. Cao et al., "Protein disulfide isomerases are promising targets for predicting the survival and tumor progression in glioma patients," Aging, vol. 12, no. 3, pp. 2347-2372, 2020.

[60] M. Shahid, K. M. Cho, M. N. Nguyen et al., "Prognostic value and their clinical implication of 89-gene signature in glioma," Oncotarget, vol. 7, no. 32, pp. 51237-51250, 2016.

[61] K.-M. Gao, X.-C. Chen, J.-X. Zhang, Y. Wang, W. Yan, and Y.-P. You, "A pseudogene-signature in glioma predicts survival," Journal of Experimental \& Clinical Cancer Research, vol. 34, no. 1, p. 23, 2015.

[62] X. Hu, E. Martinez-Ledesma, S. Zheng et al., "Multigene signature for predicting prognosis of patients with $1 \mathrm{p} 19 \mathrm{q}$ codeletion diffuse glioma," Neuro-Oncology, vol. 19, no. 6, pp. 786-795, 2017.

[63] R.-C. Chai, F. Wu, Q.-X. Wang et al., "m6A RNA methylation regulators contribute to malignant progression and have clinical prognostic impact in gliomas," Aging, vol. 11, no. 4, pp. 1204-1225, 2019.

[64] Z. Zhou, R. Huang, R. Chai et al., "Identification of an energy metabolism-related signature associated with clinical prognosis in diffuse glioma," Aging, vol. 10, no. 11, pp. 3185-3209, 2018.

[65] Y.-Q. Liu, R.-C. Chai, Y.-Z. Wang et al., "Amino acid metabolism-related gene expression-based risk signature can better predict overall survival for glioma," Cancer Science, vol. 110, no. 1, pp. 321-333, 2019.

[66] X. Sun, X. Liu, M. Xia, Y. Shao, and X. D. Zhang, "Multicellular gene network analysis identifies a macrophage-related gene signature predictive of therapeutic response and prognosis of gliomas," Journal of Translational Medicine, vol. 17, no. 1, p. 159, 2019.

[67] Y. Zhang, Y. Liu, H. Liu, Z. Zhao, F. Wu, and F. Zeng, "Clinical and biological significances of a methyltransferaserelated signature in diffuse glioma," Frontiers in Oncology, vol. 10, p. 508, 2020.

[68] F. Zeng, K. Wang, X. Liu, and Z. Zhao, "Comprehensive profiling identifies a novel signature with robust predictive value and reveals the potential drug resistance mechanism in glioma," Cell Communication and Signaling, vol. 18, no. 1, p. 2, 2020.

[69] W. Xu, Z. Liu, H. Ren et al., "Twenty metabolic genes based signature predicts survival of glioma patients," Journal of Cancer, vol. 11, no. 2, pp. 441-449, 2020.

[70] Z. Peng, C. Liu, and M. Wu, "New insights into long noncoding RNAs and their roles in glioma," Molecular Cancer, vol. 17, no. 1, p. 61, 2018.
[71] C. Hu, Y. Zhou, C. Liu, and Y. Kang, "Risk assessment model constructed by differentially expressed lncRNAs for the prognosis of glioma," Oncology Reports, vol. 40, no. 5, pp. 2467-2476, 2018.

[72] M. Zhou, Z. Zhang, H. Zhao, S. Bao, L. Cheng, and J. Sun, "An immune-related six-lncRNA signature to improve prognosis prediction of glioblastoma multiforme," Molecular Neurobiology, vol. 55, no. 7, pp. 3684-3697, 2018.

[73] G. Chen, Y. Cao, L. Zhang, H. Ma, C. Shen, and J. Zhao, "Analysis of long non-coding RNA expression profiles identifies novel lncRNA biomarkers in the tumorigenesis and malignant progression of gliomas," Oncotarget, vol. 8, no. 40, pp. 67744-67753, 2017.

[74] W. Wang, F. Yang, L. Zhang et al., "LncRNA profile study reveals four-lncRNA signature associated with the prognosis of patients with anaplastic gliomas," Oncotarget, vol. 7, no. 47, pp. 77225-77236, 2016.

[75] M. Kiran, A. Chatrath, X. Tang, D. M. Keenan, and A. Dutta, "A prognostic signature for lower grade gliomas based on expression of long non-coding RNAs," Molecular Neurobiology, vol. 56, no. 7, pp. 4786-4798, 2019.

[76] X.-Q. Zhang, S. Sun, K.-F. Lam et al., “A long non-coding RNA signature in glioblastoma multiforme predicts survival," Neurobiology of Disease, vol. 58, pp. 123-131, 2013.

[77] D. Li, J. Lu, H. Li, S. Qi, and L. Yu, "Identification of a long noncoding RNA signature to predict outcomes of glioblastoma," Molecular Medicine Reports, vol. 19, no. 6, pp. 5406-5416, 2019.

[78] P. Xia, Q. Li, G. Wu, and Y. Huang, "An immune-related lncRNA signature to predict survival in glioma patients," Cellular and Molecular Neurobiology, vol. 41, no. 2, pp. 365-375, 2021.

[79] E. W. Steyerberg and Y. Vergouwe, "Towards better clinical prediction models: seven steps for development and an ABCD for validation," European Heart Journal, vol. 35, no. 29, pp. 1925-1931, 2014.

[80] S. Anthiya, A. Griveau, C. Loussouarn et al., "MicroRNAbased drugs for brain tumors," Trends in Cancer, vol. 4, no. 3, pp. 222-238, 2018.

[81] Z. Qian, Y. Li, X. Fan et al., "Prognostic value of a microRNA signature as a novel biomarker in patients with lower-grade gliomas," Journal of Neuro-Oncology, vol. 137, no. 1, pp. 127-137, 2018.

[82] W. Chen, Q. Yu, B. Chen, X. Lu, and Q. Li, “The prognostic value of a seven-microRNA classifier as a novel biomarker for the prediction and detection of recurrence in glioma patients," Oncotarget, vol. 7, no. 33, pp. 53392-53413, 2016.

[83] W. Cheng, X. Ren, J. Cai et al., “A five-miRNA signature with prognostic and predictive value for MGMT promotermethylated glioblastoma patients," Oncotarget, vol. 6, no. 30, pp. 29285-29295, 2015.

[84] T. Ushijima and H. Suzuki, "The origin of CIMP, at last," Cancer Cell, vol. 35, no. 2, pp. 165-167, 2019.

[85] T. M. Malta, C. F. De Souza, T. S. Sabedot et al., "Glioma CpG island methylator phenotype (G-CIMP): biological and clinical implications," Neuro-Oncology, vol. 20, no. 5, pp. 608-620, 2018.

[86] A.-A. Yin, N. Lu, A. Etcheverry et al., "A novel prognostic six-CpG signature in glioblastomas," CNS Neuroscience \& Therapeutics, vol. 24, no. 3, pp. 167-177, 2018.

[87] A. A. Yin, Y. L. He, A. Etcheverry et al., "Novel predictive epigenetic signature for temozolomide in non-G-CIMP glioblastomas," Clinical Epigenetics, vol. 11, no. 1, p. 76, 2019. 
[88] S. Shukla, I. R. Pia Patric, S. Thinagararjan et al., "A DNA methylation prognostic signature of glioblastoma: identification of NPTX2-PTEN-NF- $\kappa$ B nexus," Cancer Research, vol. 73, no. 22, pp. 6563-6573, 2013.

[89] L. C. Stetson, J.-E. Dazard, and J. S. Barnholtz-Sloan, "Protein markers predict survival in glioma patients," Molecular \& Cellular Proteomics, vol. 15, no. 7, pp. 2356-2365, 2016.

[90] V. Patil and K. Mahalingam, "A four-protein expression prognostic signature predicts clinical outcome of lowergrade glioma," Gene, vol. 679, pp. 57-64, 2018.

[91] W.-Z. Gao, L.-M. Guo, T.-Q. Xu, Y.-H. Yin, and F. Jia, "Identification of a multidimensional transcriptome signature for survival prediction of postoperative glioblastoma multiforme patients," Journal of Translational Medicine, vol. 16, no. 1, p. 368, 2018.

[92] A. Etcheverry, M. Aubry, A. Idbaih et al., "DGKI methylation status modulates the prognostic value of MGMT in glioblastoma patients treated with combined radio-chemotherapy with temozolomide," PLoS One, vol. 9, no. 9, Article ID e104455, 2014.

[93] J. Xiong, Z. Bing, Y. Su, D. Deng, and X. Peng, “An integrated mRNA and microRNA expression signature for glioblastoma multiforme prognosis," PLoS One, vol. 9, no. 5, Article ID e98419, 2014.

[94] J. E. Eckel-Passow, D. H. Lachance, A. M. Molinaro et al., "Glioma groups based on 1p/19q, IDH, and TERT promoter mutations in tumors," New England Journal of Medicine, vol. 372, no. 26, pp. 2499-2508, 2015.

[95] T. Li, M. E. Forbes, G. N. Fuller, J. Li, X. Yang, and W. Zhang, "IGFBP2: integrative hub of developmental and oncogenic signaling network," Oncogene, vol. 39, no. 11, pp. 2243-2257, 2020.

[96] J. Cai, Q. Chen, Y. Cui et al., "Immune heterogeneity and clinicopathologic characterization of IGFBP2 in 2447 glioma samples," Oncoimmunology, vol. 7, no. 5, Article ID e1426516, 2018.

[97] M. Haberland, R. L. Montgomery, and E. N. Olson, "The many roles of histone deacetylases in development and physiology: implications for disease and therapy," Nature Reviews Genetics, vol. 10, no. 1, pp. 32-42, 2009.

[98] I. Y. Eyüpoglu and N. E. Savaskan, "Epigenetics in brain tumors: HDACs take center stage," Current Neuropharmacology, vol. 14, no. 1, pp. 48-54, 2016.

[99] K. B. Peters, E. S. Lipp, E. Miller et al., "Phase I/II trial of vorinostat, bevacizumab, and daily temozolomide for recurrent malignant gliomas," Journal of Neuro-Oncology, vol. 137, no. 2, pp. 349-356, 2018.

[100] A. Bradshaw, A. Wickremsekera, S. T. Tan, L. Peng, P. F. Davis, and T. Itinteang, "Cancer stem cell hierarchy in glioblastoma multiforme," Frontiers in Surgery, vol. 3, p. 21, 2016.

[101] R. G. W. Verhaak, K. A. Hoadley, E. Purdom et al., "Integrated genomic analysis identifies clinically relevant subtypes of glioblastoma characterized by abnormalities in PDGFRA, IDH1, EGFR, and NF1," Cancer Cell, vol. 17, no. 1, pp. 98-110, 2010.

[102] K. L. Mooney, W. Choy, S. Sidhu et al., "The role of CD44 in glioblastoma multiforme," Journal of Clinical Neuroscience, vol. 34, pp. 1-5, 2016.

[103] P. S. Filippou, G. S. Karagiannis, and A. Constantinidou, "Midkine (MDK) growth factor: a key player in cancer progression and a promising therapeutic target," Oncogene, vol. 39, pp. 2040-2054, 2020.
[104] M. A. J. Weterman, N. Ajubi, I. M. R. Van Dinter et al., "nmb, a novel gene, is expressed in low-metastatic human melanoma cell lines and xenografts," International Journal of Cancer, vol. 60, no. 1, pp. 73-81, 1995.

[105] G. Bao, N. Wang, R. Li, G. Xu, P. Liu, and B. He, "Glycoprotein non-metastaticmelanoma protein $\mathrm{B}$ promotes glioma motility and angiogenesis through the $\mathrm{Wnt} / \beta$-catenin signaling pathway," Experimental Biology and Medicine, vol. 241, no. 17, pp. 1968-1976, 2016.

[106] Y. Ono, S. Chiba, H. Yano et al., "Glycoprotein nonmetastatic melanoma protein B (GPNMB) promotes the progression of brain glioblastoma via $\mathrm{Na}+\mathrm{K}+$-ATPase," Biochemical and Biophysical Research Communications, vol. 481, no. 1-2, pp. 7-12, 2016.

[107] F. Szulzewsky, A. Pelz, X. Feng et al., "Glioma-associated microglia/macrophages display an expression profile different from M1 and M2 polarization and highly express Gpnmb and Spp1," PLoS One, vol. 10, no. 2, Article ID e0116644, 2015.

[108] M. Taya and S. R. Hammes, "Glycoprotein non-metastatic melanoma protein B (GPNMB) and cancer: a novel potential therapeutic target," Steroids, vol. 133, pp. 102-107, 2018.

[109] C. W. Brennan, R. G. Verhaak, A. McKenna et al., "The somatic genomic landscape of glioblastoma," Cell, vol. 155, no. 2, pp. 462-477, 2013.

[110] M. Weller, W. Wick, K. Aldape et al., "Glioma," Nature Reviews Disease Primers, vol. 1, no. 1, p. 15017, 2015.

[111] S. Keller and M. H. H. Schmidt, "EGFR and EGFRvIII promote angiogenesis and cell invasion in glioblastoma: combination therapies for an effective treatment," International Journal of Molecular Sciences, vol. 18, no. 6, p. 1295, 2017.

[112] E. Eskilsson, G. V. Røsland, G. Solecki et al., "EGFR heterogeneity and implications for therapeutic intervention in glioblastoma," Neuro-Oncology, vol. 20, no. 6, pp. 743-752, 2018.

[113] H. L. Goel and A. M. Mercurio, "VEGF targets the tumour cell," Nature Reviews Cancer, vol. 13, no. 12, pp. 871-882, 2013.

[114] N. Jhaveri, T. C. Chen, and F. M. Hofman, "Tumor vasculature and glioma stem cells: contributions to glioma progression," Cancer Letters, vol. 380, no. 2, pp. 545-551, 2016.

[115] Z. Zhang, B. Z. Cui, L. H. Wu, Q. L. Xu, Z. Wang, and B. Yang, "The inhibition effect of expressions of miR-221 and miR-222 on glioma and corresponding mechanism," Bratislava Medical Journal, vol. 115, no. 11, pp. 685-691, 2014.

[116] E. Brognara, E. Fabbri, G. Montagner et al., "High levels of apoptosis are induced in human glioma cell lines by coadministration of peptide nucleic acids targeting miR-221 and miR-222," International Journal of Oncology, vol. 48, no. 3, pp. 1029-1038, 2016.

[117] L. Xue, Y. Wang, S. Yue, and J. Zhang, "The expression of miRNA-221 and miRNA-222 in gliomas patients and their prognosis," Neurological Sciences, vol. 38, no. 1, pp. 67-73, 2017.

[118] V. Ramaswamy, M. Remke, E. Bouffet et al., "Risk stratification of childhood medulloblastoma in the molecular era: the current consensus," Acta Neuropathologica, vol. 131, no. 6, pp. 821-831, 2016.

[119] S. E. Nwaobi, V. A. Cuddapah, K. C. Patterson, A. C. Randolph, and M. L. Olsen, "The role of glial-specific Kir4.1 in normal and pathological states of the CNS," Acta Neuropathologica, vol. 132, no. 1, pp. 1-21, 2016. 
[120] G. Qin, X. Li, Z. Chen et al., "Prognostic value of YKL-40 in patients with glioblastoma: a systematic review and metaanalysis," Molecular Neurobiology, vol. 54, no. 5, pp. 3264-3270, 2017.

[121] K. M. P. Batista, S. A. D. Eulate-Beramendi, K. Y. Á. R. D. Pińa et al., "Mesenchymal/proangiogenic factor YKL-40 related to glioblastomas and its relationship with the subventricular zone," Folia Neuropathologica, vol. 55, no. 1, pp. 14-22, 2017.

[122] K. Swiatek-Machado and B. Kaminska, "STAT signaling in glioma cells," Advances in Experimental Medicine and Biology, vol. 986, pp. 189-208, 2013.

[123] L. A. Kluth, P. C. Black, B. H. Bochner et al., "Prognostic and prediction tools in bladder cancer: a comprehensive review of the literature," European Urology, vol. 68, no. 2, pp. 238-253, 2015.

[124] A. Bradley, R. Van Der Meer, and C. J. McKay, “A systematic review of methodological quality of model development studies predicting prognostic outcome for resectable pancreatic cancer," BMJ Open, vol. 9, no. 8, Article ID e027192, 2019.

[125] T. Klatte, S. H. Rossi, and G. D. Stewart, "Prognostic factors and prognostic models for renal cell carcinoma: a literature review," World Journal of Urology, vol. 36, no. 12, pp. 1943-1952, 2018.

[126] A. L. Mahar, C. Compton, S. Halabi, K. R. Hess, M. R. Weiser, and P. A. Groome, "Personalizing prognosis in colorectal cancer: a systematic review of the quality and nature of clinical prognostic tools for survival outcomes," Journal of Surgical Oncology, vol. 116, no. 8, pp. 969-982, 2017.

[127] M. T. Phung, S. Tin Tin, and J. M. Elwood, "Prognostic models for breast cancer: a systematic review," BMC Cancer, vol. 19, no. 1, p. 230, 2019.

[128] K. M. Veen, I. B. De Angst, M. M. Mokhles et al., "A clinician's guide for developing a prediction model: a case study using real-world data of patients with castration-resistant prostate cancer," Journal of Cancer Research and Clinical Oncology, vol. 146, no. 8, pp. 2067-2075, 2020.

[129] G. Xiao, X. Zhang, X. Zhang et al., "Aging-related genes are potential prognostic biomarkers for patients with gliomas," Aging, vol. 13, no. 9, pp. 13239-13263, 2021.

[130] A. J. Vickers and E. B. Elkin, "Decision curve analysis: a novel method for evaluating prediction models," Medical Decision Making, vol. 26, no. 6, pp. 565-574, 2006.

[131] A. Van Giessen, J. Peters, B. Wilcher et al., "Systematic review of health economic impact evaluations of risk prediction models: stop developing, start evaluating," Value in Health, vol. 20, no. 4, pp. 718-726, 2017. 